\title{
El uso de los sistemas tradicionales de organización del conocimiento en las bibliotecas peruanas
}

\author{
Rosa Dorival Córdova \\ Universidad del Pacífico - Perú \\ Carlos Javier Rojas Lazaro \\ Universidad Nacional Mayor de San Marcos - Perú
}

\section{ANÁLISIS / ANALYSIS}

\section{Resumen}

Se describe y analiza el uso de los sistemas tradicionales de organización del conocimiento, como por ejemplo, los sistemas de clasificación decimal Dewey (SCDD) y de la Library of Congress (LC), las listas de encabezamientos de materia (LEMB) y los tesauros; en las unidades de información peruanas. Asimismo, se identifican los tesauros elaborados en el Perú desde la década de los años 70 del siglo pasado a la fecha.

Palabras clave

Sistemas tradicionales de organización del conocimiento; Sistemas de clasificación; Listas de encabezamientos de materia; Tesauros; Lenguajes documentales; Perú

\section{The use of traditional systems of knowledge organization in peruvian libraries}

\section{Abstract}

It describes and discusses the use of the most traditional Systems of Knowledge Organization, such as the Dewey Decimal Classification and the Library of Congress (LC), lists of subject headings and thesauri; in the Peruvian information systems. It also identifies the thesauri produced in Peru since the decade of the seventies of the last century to date.

Keywords

Systems of Knowledge Organization (SKO); Clasification system; Subject headings; Thesaurus; Indexing languages; Peru

\section{Introducción}

Desde los inicios de la formación de las bibliotecas, el bibliotecario se ha valido de diversos sistemas, instrumentos o herramientas que le han permitido organizar la información y las colecciones que le son confiadas. Estos son los sistemas de organización del conocimiento (SOC). En la actualidad, existen los llamados de uso tradicional, como los sistemas de clasificación, las listas de epígrafes, los tesauros, entre otros; y los más modernos que han aparecido de la mano de las nuevas tecnologías y de Internet, como las ontologías, taxonomías, redes semánticas, etc.

Además de su importancia en la organización del conocimiento, los SOC juegan un papel fundamental como intermediarios entre la demanda de los usuarios y la colección. En este sentido son lenguajes de búsqueda 
esenciales para la recuperación de información. En esta oportunidad, hacemos un análisis desde el punto de vista del bibliotecario y no del usuario.

En este ensayo no vamos a discutir cuál de estos sistemas es el mejor ya que estamos convencidos que si un sistema está bien aplicado y cumple eficazmente su función, entonces es un buen sistema. Además, no hay que olvidar que la aplicación de uno u otro sistema dependen de las características de cada unidad de información.

En el Perú existe una disparidad en el desarrollo de las unidades de información (UI). Encontramos, por un lado, UI tecnológicamente muy avanzadas y de otro lado, Ul que sufren de escasez de recursos, totalmente relegadas y que se ven obligadas al uso poco eficiente de herramientas tradicionales.

Sobre este último tema debemos señalar que, en el Perú, la elección de los sistemas a utilizar generalmente no es parte de una evaluación al momento de diseñar la organización de la biblioteca. Se decide por la herramienta que se tiene a mano o bien que no exija gastos adicionales a nivel presupuestal.

\section{Sistemas de Clasificación y Listas de Encabezamientos de Materia}

Podemos señalar que la gran mayoría de unidades de información en el Perú trabajan con sistemas tradicionales de organización del conocimiento: El sistema de clasificación decimal Dewey, con la modalidad de estantería abierta, y la Lista de encabezamientos de materia para bibliotecas (LEMB).

El uso en el Perú de los SOC tradicionales antes mencionados, se debe, en primer lugar, a sus características propias: La universalidad o generalidad del sistema Dewey y de las LEMB los hacen muy útiles y prácticos para cualquier tipo de unidad de información. En el caso del Sistema Dewey, su estructura jerárquica -o en forma de árbol- apoya la organización sistemática del conocimiento y proporciona un ordenamiento lógico al agrupar los conceptos específicos dentro de los conceptos generales, lo que facilita la tarea de organizar la colección en la estantería. El agrupar las materias en grandes categorías y sus niveles permite tener, además, una idea global de la colección.

En cuanto a la LEMB, la estructura asociativa o combinatoria que presentan los conceptos al organizar los términos de manera alfabética y con la posibilidad de combinarlos entre sí, permite que hasta la unidad de información más pequeña pueda extraer temas con suma facilidad.

Además de sus beneficios internos, los SOC tradicionales han sido ampliamente difundidos en nuestro medio, están en castellano y son de fácil accesibilidad. Las capacitaciones proporcionadas por la Biblioteca Nacional del Perú han permitido que el sistema Dewey, en sus grandes clases, se encuentre muy difundido; $y$, en ese sentido, es mayor la familiaridad de los usuarios con este sistema.

En el Perú se utilizan, en menor proporción, los tesauros -que se aplican sobre todo en bibliotecas especializadas-, el sistema de clasificación de la Library of Congress (LC) -en bibliotecas universitarias- y el sistema facetado; este último en mucha menor medida, aunque tuvo un auge importante en las organizaciones no gubernamentales (ONG) durante la década de los 80 del siglo pasado.

Es importante mencionar a los sistemas propios usados en muchas unidades de información pequeñas del interior del país, donde la clasificación es correlativa y la indización temática es por grandes temas. De estos usos existe una gran variedad de combinaciones.

Son muchos los dolores de cabeza de los especialistas en procesos técnicos, en particular y de los bibliotecólogos, en general, al enfrentarse a la tarea cotidiana de indizar. Aquí queremos referirnos específicamente a dos problemas ampliamente conocidos, tanto en el sistema Dewey como en la LEMB: la desactualización de los temas y la cada vez más lejana relación con la realidad peruana.

En un estudio desarrollado por MacEwan (1998), se señala como desventajas en el uso de la LC en otros países, particularmente de América Latina, los siguientes puntos: 
1. El empleo de "americanismos".

2. La perspectiva político-cultural de los Estados Unidos de América.

3. La permanencia de términos anticuados.

4. El enfoque occidental en el tratamiento de los asuntos religiosos y étnicos.

5. La falta de especificidad del vocabulario.

Cuántos de nosotros hemos tenido que dejar un libro por algunos días al no poder asignarle un número de clasificación, al no poder identificar un término en la lista de encabezamientos; o el tener que buscar especialistas o consultar con colegas para decidir con mucha seguridad los temas. La tendencia es a extraer y usar las diferentes expresiones de los conceptos directamente de la fuente, generando una diversidad temática muy diversa.

Esto se presenta debido a que los sistemas tradicionales son sistemas controlados y coordinados; es decir, que en éstos se elabora con anterioridad una lista cerrada y normativa de términos o símbolos. Se construyen a priori con un vocabulario predefinido cuyos términos son los únicos aceptados.

Los lenguajes controlados tienen la ventaja de normalizar los catálogos, ya sean manuales o automatizados y permiten a los usuarios evitar enfrentarse a problemas de sinonimia, homonimia y otros. La desventaja está en su rigidez, ya que hacer modificaciones implica crear una lista propia por cada unidad de información.

En este sentido, es grande la cantidad de términos que se deben generar y que son propios de cada país e incluso de cada localidad. Por ejemplo, los términos regionalización o descentralización, violencia política, entre otros, no existen ni en el sistema Dewey ni en la LEMB.

Asimismo, son pocas las referencias a la realidad latinoamericana en el sistema Dewey. Por ejemplo, la parte de Historia en este esquema es muy simple y escueta; más aún, la clase Literatura ha ubicado a todas las naciones latinoamericanas bajo un mismo número, para lo cual hay que agregar un sufijo de país para diferenciarlos. Las referencias "racistas" a etnias obligan, también, a crear términos adecuados y que se correspondan con el desarrollo del lenguaje. Existe también la desactualización en cuanto al desarrollo de las ciencias, como lo prueba la no existencia de términos como Neurociencia, Inteligencia Emocional, entre muchos otros.

Tanto el sistema de clasificación decimal Dewey como la lista de encabezamientos de materia se ven complementados y apoyan la organización de la información cuando la unidad de información organiza su colección con estantería abierta. Luego que el usuario ha "buceado" por el fichero, ya sea manual o automatizado, y siente que no ha podido hallar lo que necesita, un paseo por la estantería le resultará refrescante y muy útil.

\section{Tesauros}

Los antecedentes documentales del concepto tesauro los encontramos en el tesauro lingüístico Thesaurus of English Words and Phrases, obra de Peter Mark Roget, publicado en 1852. Vickery (1960), citado por Iglesias (1997, p. 42), señala que la primera persona que utiliza documentalmente la palabra "tesauro", desde la óptica de la recuperación de la información, es Helen Brownsen; quien, en 1957 y en una conferencia sobre clasificación realizada en Dorking, Inglaterra, dijo:

[...] el problema [de la recuperación de la información], al menos como algunos investigadores lo ven, es transformar los conceptos y sus relaciones como aparecen expresadas en el documento a otro lenguaje más reglamentado, con los sinónimos controlados y las estructuras sintácticas simplificadas [...] Algunos investigadores piensan que la mejor respuesta o solución [...] puede ser la aplicación de un tesauro mecanizado basado en redes o significados relacionados (Vickery, 1960). 
Después de la Segunda Guerra Mundial, particularmente en Europa y el mundo anglosajón, se produce un creciente aumento de las publicaciones científicas y técnicas, generando que la utilización de los sistemas enciclopédicos de tratamiento y recuperación de información presenten ineficacia en el tratamiento de las publicaciones especializadas de uso en bibliotecas académicas y especializadas.

El tesauro es un lenguaje postcoordinado de estructura combinatoria, vocabulario controlado y especializado por naturaleza; pese a que, según muchos autores, no nació con la idea de postcoordinación. Surge más bien con la noción básica de los encabezamientos de materia, que es un lenguaje precoordinado. No es hasta mediado del siglo XX que el concepto de tesauro se desarrolla, coincidiendo con la teoría de la coordinación de conceptos y el sistema de unitérminos, creado por Mortimer Taube en 1952. Posteriormente se consolida con el desarrollo tecnológico y, particularmente, de la informática, con la aparición de las computadoras.

El tesauro es un tipo de vocabulario controlado que facilita la comunicación y la transferencia de información, al eliminar la ambigüedad del lenguaje natural y libre en que está escrito un documento; por lo tanto, enfatiza los conceptos sobre las palabras. Permite un control sobre los términos sinónimos y homógrafos, mediante relaciones de equivalencia (USE y UP). Asimismo, muestra todos los términos relevantes, que posiblemente el usuario no imaginó -por ejemplo, a un tema en general o particular conceptualizado-, por medio de términos genéricos (TG), términos específicos (TE) y los términos asociados o relacionados (TR). Entonces podemos concluir que el tesauro es un tipo de lenguaje documental que hace interactuar las ideas del autor (documento), el analista de información documental (indizador) y las necesidades de información de las personas (usuarios).

Peón (2005, p. 107-108), señala que la elección de un lenguaje documental postcoordinado -como el tesauro- para una disciplina es sencilla debido a las siguientes consideraciones fundamentales:

- La primera de ellas es la simple valoración del tesauro como lenguaje documental en cuanto a su tipología postcoordinada que ofrece las posibilidades combinatorias de descripción y recuperación de las informaciones requeridas.

- La segunda es la de la pluralidad estructural de este tipo de lenguajes documentales en cuanto a su composición sobre la base de una Parte Alfabética y una parte Sistemática, lo que posibilita conjugar en su utilización y uso las distintas posibilidades que brindan los lenguajes de estructura asociativa y los lenguajes de estructura jerarquizada, a lo que hay que añadir las posibilidades de localización directa de términos y conceptos que ofrece en múltiples ocasiones la presencia añadida de un Índice Permutado.

- La tercera consideración es la de la utilización por parte de los Tesauros como elementos de descripción de términos controlados del lenguaje natural, a los que se acompaña una codificación identificativa en la Parte Sistemática, por lo que ofrecen un alto grado de fiabilidad en el empleo de sus términos.

- En cuarto lugar se debe considerar como un criterio fundamental de valorización la especial idoneidad de los Tesauros para su desarrollo y aplicación a campos temáticos y áreas específicas de conocimiento, dado que la estructura plural de su conformación se adapta muy adecuadamente a campos temáticos concretos permitiendo la introducción complementaria de elementos descriptores de otras disciplinas.

En el Perú, los tesauros han sido utilizados desde la década de los 70 del siglo pasado por las bibliotecas especializadas; muchas instituciones limeñas tuvieron que elaborar sus tesauros debido a que en su especialidad no había información relevante sistematizada, como por ejemplo:

- 1976, Tesauro ITINTEC, elaborado inicialmente por el Instituto de Investigación Tecnológica Industrial y de Normas Técnicas (ITINTEC). A la fecha, existe la $2^{\underline{a}}$ edición publicada en 1985 con el título Tesauro de terminología técnica, edición corregida y aumentada por Isabel Montoya Ramírez del Villar. 
- 1978, Tesauro de Términos de la Integración Subregional Andina, elaborado por la Biblioteca de la Junta del Acuerdo de Cartagena, hoy Comunidad Andina de Naciones; en 1979, se publicó la 2ª edición, y en 1983 la 3ª Edición. Este documento se inició en julio de 1975 con el nombre de Tesauro de Términos de Integración Andina (TTIA), especializado en integración económica. En 1990, se modifica el título a Microtesauro de integración y términos relacionados al proceso de integración andino.

- 1982, Tesauro de la educación: Área tecnología educativa, elaborado por Nelly MacKee, Danilo Ordóñez B. y Aurora de la Vega y publicado por el Instituto Nacional de Investigación y Desarrollo de la Educación (INIDE).

- 1982, Tesauro de ingeniería sanitaria y ciencias del ambiente, elaborado inicialmente por la Red Panamericana de Información y Documentación en Ingeniería Sanitaria y Ciencias del Ambiente (REPIDISCA), dependiente del CEPIS, que actualmente va por la 17 edición, del año 2004, denominada Tesauro de ingeniería sanitaria y ambiental.

- 1984, Tesauro de trabajo social, elaborado por la bibliotecóloga María Inés Dávila Pessagno y publicado por el CELATS; en el año 2005, Rojas Eberhard Editores, con sede en la ciudad de Bogotá, D.C., ha publicado la $2^{\mathrm{a}}$ edición actualizada y ampliada por la misma autora.

- 1990, Microtesauro de integración y términos relacionados al proceso de integración andino, elaborado por el Centro de Documentación de la Junta del Acuerdo de Cartagena, basado en el TTIA. La versión preliminar se publicó en 1987.

- 1991, Tesauro de tecnologías apropiadas, elaborado por la bibliotecóloga María Antonieta Ballón Delgado y publicado por el Instituto de Transferencias de Tecnologías Apropiadas del Convenio Andrés Bello (ITACAB).

- 1992, Lista de descriptores de RIALIDE, elaborado por el Centro de Documentación de la Asociación Latinoamericana de Instituciones Financieras de Desarrollo (ALIDE), coordinador regional de la Red de Información de ALIDE (REALIDE); en este año puso a disposición de sus integrantes una versión actualizada de la $4^{a}$ edición de 1991. Su estructura era compatible con el Macrothesaurus para el procesamiento de la información relativa al desarrollo económico y social de las Naciones Unidas y la OCDE.

- 1998, Thesaurus sobre reforma del sector salud, elaborado por la División de Desarrollo de Sistemas y Servicios de Salud (HSP) de la Organización Panamericana, OMS, en el marco del Proyecto de Sistema de Información y Análisis OPS/AID para la región de las Américas.

- 2004, Tesauro de ingeniería sanitaria y ambiental, elaborada y actualizada por la Biblioteca Virtual de Desarrollo Sostenible y Salud Ambiental de la OPS, disponible en: htpp://www.cepis.ops-oms.org/bvsair/e/manuales/tesa/teses.pdf. Documento basado en el Tesauro de ingeniería sanitaria y ciencias del ambiente.

- La Universidad de León (s.f., Período científico, para. 17-19), a través de su Área de Biblioteconomía y Documentación, señala, que luego del nivel alcanzado por el tesauro debido a los estudios de semántica y sintaxis documentales, lo siguiente:

La gran euforia con que fue acogido el tesauro se convertiría en cierto desencanto ante la gran proliferación y tipología que alcanzaron, lo que implicó que se fueran levantando auténticas barreras lingüísticas documentales que dificultan la transmisión rápida de la información entre los centros. Actualmente, existe una tendencia a buscar el equilibrio perdido ante tan excesiva especialización y tipología lingüística documental y se observa una vuelta a los lenguajes documentales más generales .... Esta vuelta a la universalidad no implica en absoluto un regreso a la rigidez estructural de los lenguajes jerárquicos, sino una vuelta al enciclopedismo temático, aunque éste sea restringido, con otra proyección estructural. 
Moreiro y Méndez (1999, p. 20) enfatizan que "La gestión del conocimiento, que es la tendencia de todos los sistemas de información actuales, no tratan de crear un simple almacenamiento y acceso a la información, sino todo un proceso de manipulación, selección, mejora y preparación de la información, para dotarla de un valor añadido”.

\section{Conclusiones y recomendaciones}

A modo de conclusión, podemos decir que estos SOC tradicionales ofrecen a su vez ventajas y retos para los bibliotecólogos peruanos; sin embargo, deberían ser los entes rectores quienes convoquen a un grupo multidisciplinario permanente para:

- Delinear una política de indización nacional en base a principios internacionales.

- Uniformizar el lenguaje a fin de eliminar la diversidad de vocabularios y clasificaciones, sin olvidar las bibliotecas de comunidades nativas y quechua hablantes. Elaborar listas de autoridades de materia.

- Generar un léxico general de términos - peruanos- basados en los sistemas ya establecidos, los cuales deberán ser revisados y actualizados, adoptando, traduciendo o adaptando los términos que más se ajusten a nuestra realidad.

\section{Referências}

Bermello, L. (2001). Tendencias de desarrollo de los lenguajes de encabezamientos de materia en las bibliotecas. Acimed, 9(2), 109-120. Recuperado de http://bvs.sld.cu/revistas/aci/vol9 2 02/aci03201.pdf

Currás, E. (2005). Ontologías, taxonomías y tesauros: manual de construcción y uso. Gijón, Asturias: Ediciones Trea.

Gimeno, J. (2002). Sistemas de indización aplicados en bibliotecas: clasificaciones, tesauros y encabezamientos de materia. En J. A. Magán (Coord.). Tratado básico de biblioteconomía (pp. 199-229). Madrid: Editorial Complutense.

Iglesias, M. T. (1997). Beneficios de la utilización de lenguajes controlados en el análisis y recuperación de información. Investigación Bibliotecológica, 11(23), 32-45.

Jiménez, J. E. (1998). Consideraciones sobre la indización en las bibliotecas universitarias españolas. En FESABID 98: VI Jornadas Españolas de Documentación. Madrid. Recuperado de http://fesabid98.florida-uni.es/Comunicaciones/i elias.htm

MacEwan, A. (1998). Working with LCSH: the cost of cooperation and the achievement of access. A perspective from the British library. Amsterdam.

Márzal, M., Beltrán, P., Morato, J. L., Llorens, J., Moreira, J. A. \& Sánchez, S. (2002). Propuesta para la utilización de estructuras verbales aplicadas a la recuperación y representación de la información. Scire, 8(1), 95-102.

Moreiro, J. A. y Méndez, E. M. (1999). Lenguaje natural e indización automatizada. Ciencias de la Información, 30(3), 11-24.

Peón, J. L. (2005). Lenguajes documentales y administración pública: análisis de tesauros en lengua española. Revista General de Información y Documentación, 15(1), 105-122.

Soto, S. (2000). La recuperación de la información: ¿Lenguaje natural vs. Lenguaje controlado? En Argentina. Instituto Nacional de la Administración Pública, INAP, Eds. Proceedings Seminario Dilemas de la Biblioteca Actual, pp. 1-7, Buenos Aires. Recuperado de http://eprints.rclis.org/archive/00006029/

Universidad de León. Área de Biblioteconomía y Documentación. (s.f.). Historia de los lenguajes documentales. Recuperado de http://www3.unileon.es/dp/abd/tesauro/pagina/conceptos/historia.htm 


\section{Datos de los autores}

\section{Rosa Dorival Córdova}

Licenciada en Sociología, egresada de la Escuela Nacional de Bibliotecarios, egresada de la Maestría en Bibliotecología e Información de la Universidad Nacional Mayor de San Marcos. Ha publicado libros y artículos sobre bibliografías especializadas en temas afroperuanos y de ciencias sociales. Actualmente, esta a cargo de la Biblioteca de la Universidad del Pacífico, así como abocada a trabajos de promoción de la lectura tanto en Lima como en el interior del país y en la investigación sobre afroperuanos y discriminación en el Perú.

dorival re@up.edu.pe

\section{Carlos Javier Rojas Lazaro}

Bibliotecólogo. Bachiller y licenciado por la Universidad Nacional Mayor de San Marcos (UNMSM). Desde 1993 es funcionario de la Biblioteca Nacional del Perú (BNP); actualmente, es Director Ejecutivo de Procesos Técnicos Hemerográficos. Desde 1998 es docente auxiliar del departamento académico de Bibliotecología y Ciencias de la Información de la UNMSM en el Área de Procesamiento de la Información. Ha publicado diversos documentos en su especialidad. Es expositor en eventos a nivel local, nacional e internacional. En el 2004 ha participado en la Segunda Reunión IFLA de Expertos sobre un Código Internacional de Catalogación, realizada en Buenos Aires, Argentina. En el 2005 participó como expositor en el Primer Encuentro Internacional de Catalogadores, desarrollado en Lima, Perú, en representación de la BNP y de la UNMSM.

carlosrojas12@gmail.com

Recibido - Received: 2012-01-29

Aceptado - Accepted: 2012-03-30

\section{(c) EY-NC-ND}

This work is licensed under a Creative Commons

Attribution-Noncommercial-No Derivative Works 3.0 United States License.

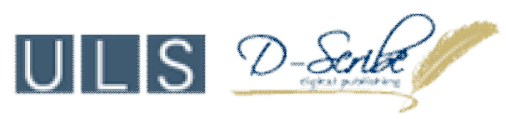

This journal is published by the University Library System of the University of Pittsburgh as part of its $\underline{D-S c r i b e}$ Digital Publishing Program and is cosponsored by the University of Pittsburgh Press. 